\title{
Enhancing Evaluation of Potential Romantic Partners Online
}

Doug Zytko

New Jersey Institute of Technology

Newark, NJ 07102, USA

daz2@njit.edu

Permission to make digital or hard copies of all or part of this work for personal or classroom use is granted without fee provided that copies are not made or distributed for profit or commercial advantage and that copies bear this notice and the full citation on the first page. Copyrights for components of this work owned by others than ACM must be honhts Abstrating with credit is permitted. To copy otherwise, or republish, to post on with credis is permitted. To copy otherwise, or republist A post on servers or to redistribute to lists, requires permission and/or a fee. Requ

from Permissions@acm.org.

2016, Sanibel Island, FL, USA

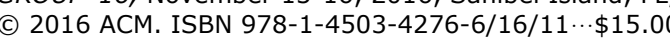

DOI: http://dx.doi.org/10.1145/2957276.2997030

\begin{abstract}
Online dating systems are a common way to discover romantic partners. Yet there persists a gap in knowledge regarding how users of these systems determine which potential partners are worthy of inperson meetings, as well as the outcomes of these inperson meeting decisions. The objective of this dissertation is two-fold: 1 ) to understand how online dating system users make decisions to meet or not meet potential romantic partners in-person, and 2) to understand how online dating system designs currently support-and could better support-predictions of initial in-person attraction to potential romantic partners.
\end{abstract}

\section{Author Keywords}

Online dating; impression formation; social matching

\section{ACM Classification Keywords}

H.5.m. Information interfaces and presentation (e.g., $\mathrm{HCI}$ ): Miscellaneous

\section{Research Questions}

The dissertation explores four main research questions:

RQ1. How do online dating system users choose which potential partners to meet in-person and which to not meet (i.e. disqualify from further evaluation)? 
RQ2. Do users make the right decisions over which potential partners to meet in-person based on information they gather?

RQ2a. Do users gather enough information to make adequately informed in-person meeting decisions?

RQ3. What is the relationship between the various commercial online dating systems and their features, and in-person meeting decision success?

RQ4. How can online dating systems be designed to better support evaluation of potential partners, so that they are more satisfied with and confident about their in-person meeting decisions?

These research questions are examined through four studies (two of which are completed), associated theory building and testing, and software design and development.

\section{Completed Work}

Study 1 - Qualitative exploration of the online dater evaluation process from the user's perspective [e.g. 3]; Research questions examined: 1, 2, \& 3

Prior online dating studies typically looked at isolated aspects of user evaluation (e.g. detecting deception in profile pages) rather than how users consolidate all the information available to them in these systems to make in-person meeting decisions. Goals of this first study were to gain a broad understanding of how active online dating system users make in-person meeting decisions, as well as the "success" of these decisions [5]. Additionally, the study aimed to probe users' selfpresentation strategies to explore how impression management motives may affect evaluations of potential partners. This study was conducted through semi-structured interviews with 41 active users of the online dating system OkCupid. Results showed that participants considered profile pages a key determinant of which potential partners were worthy of a messaging conversation, but the messaging conversation was the main determinant in the decision to meet in-person. Participants' first dates typically did not result in a second date because of online impressions deemed inaccurate in-person. These inaccuracies typically regarded personality traits rather than aspects of physical appearance or objective demographic qualities. While prior attraction literature would suggest interaction is an effective way to evaluate personality, users in this study employed OkCupid's messaging interface more so to enact thinly-veiled selfpresentation and evaluation scripts rather than to have conversations.

Study 2 - Qualitative exploration of the online dater evaluation strategies of online dating coaches [e.g. 4]; Research questions examined: $1,2, \& 3$

Interviewing active users of online dating systems, as in study 1 , is an effective way to discover user struggles with the online dater evaluation process, but not so much for successful evaluation strategies. Active users of online dating systems presumably have had less success achieving long-term romantic relationship goals, otherwise they would no longer be active users of these systems. The objective of study 2 was to garner an understanding of online dater evaluation strategies that yielded successful in-person meeting decisions. This objective was pursued through semistructured interviews with online dating coaches-selfproclaimed online dating experts who teach strategies for how to use online dating systems to successfully achieve a particular relationship goal. Results from this 
Design Requirements for Problem-Solving Discussion in Online Dating Systems

\section{- Provide conversation topics}

\section{of personal relevance: users}

may lose interest in debating a problem-solving topic that does not apply to their personal situation. Topics about common dating conflicts (e.g. "who should pay on the first date?") may maintain a focus on the conversation.

\section{- Procure users' opinions on}

\section{the topic before}

conversation: due to users'

impression management motives, they may be tempted to agree with their partner's opinion by default to save face. Procuring users' opinions prior to learning each other's opinions may prevent this.

\section{- Require resolution of}

\section{conflicting opinions: to}

prevent users from shifting the conversation to alternative topics (e.g. their hobbies, attractive qualities), the system can require users to explicate a mutually agreed upon decision at the end of conversation. Minimum conversation lengths can be enforced as well. study showed that online dating coaches considered accurate evaluation of potential romantic partners to be unlikely in today's online dating systems. Specifically, they considered evaluation of personality compatibility to be fundamentally stifled by users' poor abilities to deliberately self-present their own personalities coupled with their impression management motivations. The coaches instead promoted strategies that can yield inperson dates quickly with potential partners that satisfy minimal evaluation criteria, such as through copy-andpasted message content that is sent to hundreds of potential partners in succession. The coaches explained that first dates procured for or by clients with their strategies seldom lead to second dates, but the high frequency of dates counteracts this likely failure.

Theoretical Contribution Informed by Initial Studies

Findings from studies 1 and 2 demonstrate online daters' struggles with predicting in-person personality compatibility and thus initial romantic attraction to potential partners, which leads to unsuccessful inperson meeting decisions. While attraction literature positions interaction as a prime way to evaluate personality compatibility, studies 1 and 2 revealed online dater strategies that leveraged online interaction primarily for overt self-presentation and evaluation strategies.

The literature does suggest how people can effectively evaluate or experience personality compatibility. Prior work from the 1970s has shown that "problem-solving discussions" amongst married couples can predict marital satisfaction at current and future times [e.g. 2]. "Problem-solving discussions" [1, page 5] are those in which two partners contend with differences of opinion to reach consensus on a topic (e.g. "which spouse should take out the trash?").
Models resulting from this work [1] posit that this predictive power is due to personality attributions that married partners make regarding statements expressed in such discussions-these discussions serve as vessels of concentrated personality expression. From this it follows that problem-solving discussions could potentially help online daters (potential romantic partners) predict personality compatibility and thus romantic attraction across modalities in the same way that these discussions have predicted relationship satisfaction across time. The prior research involving problem-solving discussions between married couples [e.g. 1, 2], in combination with the findings from my prior studies, will be used to inform design

requirements for facilitating problem-solving interaction in online dating systems. Some design requirements derived from studies 1 and 2 are summarized in the side bar.

\section{Work in Progress}

Study 3 - Quantitative study of how system design predicts in-person meeting decision success

Research questions examined: 3

While studies 1 and 2 are some of the first to provide insight into how users make in-person meeting decisions and the outcomes of their in-person meeting decisions, the qualitative nature of those studies prevents us from testing associations between specific elements of online dating systems and in-person meeting outcomes. This study serves as a quantitative counterpart to the first two studies. More specifically, the objective of this study is to investigate how popular online dating systems and the interface components that comprise their design are associated with success of in-person meeting decisions. Such interface components include profile page content, use of messaging interfaces, and tools that facilitate the quantity of potential partners 


\section{Variables for Study 4}

Independent variable: type of messaging interface used by a pair of potential partners

Condition 1: no messaging Condition 2: unprompted messaging interface Condition 3: A prompted messaging interface; the conversation starts with agreement on the topic Condition 4: A prompted messaging interface; the conversation starts with disagreement on the topic (i.e. problem-solving discussion)

\section{Dependent variables:}

- Accuracy and confidence of personality trait prediction

- Accuracy and confidence of personality compatibility prediction

- Accuracy and confidence of romantic attraction prediction

- Accuracy and confidence of prediction of desire to meet the potential partner for a second meeting that are discovered and contactable in the system (e.g. Tinder's swiping interface). This study's objective will be pursued through a survey study of online daters that inquires about the last online dating system user that they met in-person and the outcome of the first meeting with that user.

Study 4 - Technology intervention to enhance inperson meeting decision success Research questions examined: 4

The final study entails a large-scale experiment in which a minimum 200 potential romantic partners are observed as they evaluate each other online through profile pages and messaging conversations, and then validate their evaluations in-person. Speed dating events organized through meetup.com will be used as a recruitment method and context for the initial in-person meetings between participants. Aside from being the first study to examine the online dater evaluation process from the perspective of both users involved, the experiment intends to investigate if text-based messaging conversations structured around problemsolving topics can facilitate more accurate predictions of personality compatibility in-person, romantic attraction in-person, and desires for a second in-person meeting than unstructured messaging conversations. The independent variable in the study is the type of messaging interface used by a pair of potential partners. The dependent variables entail various dimensions of online dater evaluation accuracy and participants' confidence in their predictions.

Variables are summarized in the sidebar.

\section{Expected Contributions}

This research is important because it bridges several research disciplines including evolutionary biology, social psychology, and human-computer interaction.
The proposed research plan has and will continue to produce findings about how people evaluate and interact with new acquaintances online for a variety of integral social needs like romance [5] as well other social desires [e.g. 4]. Expected contributions of this dissertation include a theoretical model of the process of evaluating potential romantic partners discovered in online dating systems, an understanding of online dating system users' evaluation strategies through all stages of the online dater evaluation process, and innovative design concepts for better facilitating evaluation of potential romantic partners in online dating systems as well as other social matching and social discovery domains in which in-person meetings are a goal of users.

\section{References}

1. Benjamin R. Karney and Thomas N. Bradbury. "The longitudinal course of marital quality and stability: A review of theory, methods, and research. Psychological bulletin 118.1 (1995): 3-34.

2. Howard J. Markman. 1979. Application of a behavioral model of marriage in predicting relationship satisfaction of couples planning marriage. Journal of Consulting and Clinical Psychology 47.4 (1979): 743-749.

3. Doug Zytko, Guo Freeman, Sukeshini A. Grandhi, Susan C. Herring, and Quentin Gad Jones. 2015. Enhancing evaluation of potential dates online through paired collaborative activities. In

Proceedings of the 18th ACM Conference on Computer Supported Cooperative Work \& Social Computing, 1849-1859.

4. Douglas Zytko, Sukeshini A. Grandhi, and Quentin Jones. 2014. The coaches said...what? Analysis of online dating strategies recommended by dating coaches. In Proceedings of the 19th International Conference on Supporting Group Work, in press. 